\title{
Stacked Tantalum Cones as a Method for Treating Severe Distal Femoral Bone Deficiency in Total Knee Arthroplasty
}

\author{
Ashok Rajgopal, MS, MCh, FRCS ${ }^{1} \quad$ Inayat Panda, MS, DNB ${ }^{1} \quad$ Sidharth Yadav, MS ${ }^{1}$ Om Wakde, MS, DNB ${ }^{1}$ \\ ${ }^{1}$ Department of Orthopaedics, Fortis Bone and Joint Institute, Fortis \\ Escorts, New Delhi, India \\ Address for correspondence Ashok Rajgopal, MS, MCh, FRCS, Fortis \\ Bone and Joint Institute, Fortis Escorts, Okhla Road, New Delhi \\ 110025, India (e-mail: a_rajgopal@hotmail.com). \\ J Knee Surg 2019;32:833-840.
}

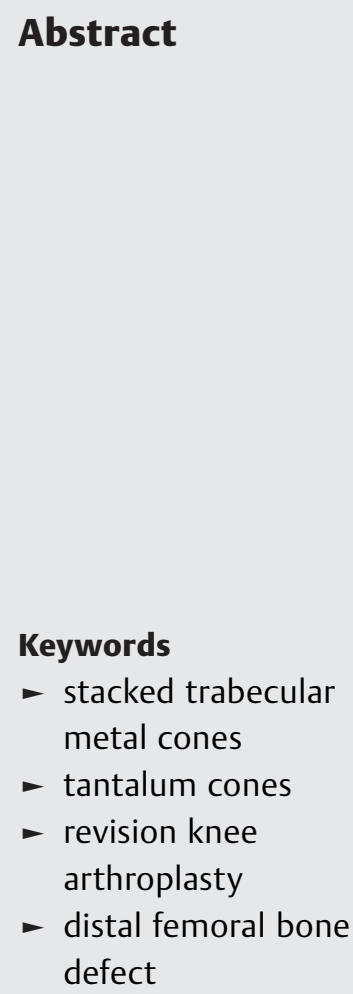

Severe bone loss in distal femur has traditionally been managed with bulk cementing, bone graft, metal augments, trabecular metal cones, and mega prosthesis. The authors present an alternative method using a stacked configuration of two tantalum cones to manage severe distal femoral bone loss. This study aims to evaluate the mid-term outcomes and possible complications in patients treated with this novel technique. We retrospectively analyzed the prospectively maintained records of 16 knees (in 16 patients) presenting with severe distal femoral bone deficiency due to septic loosening (5), giant cell tumor (GCT; 4), periprosthetic fracture (3), aseptic loosening (3), and distal femoral comminuted fracture with severe osteoarthritis (1). A standard medial parapatellar approach was taken in all our cases followed by preparation of distal femoral bone defect and use of two bridging tantalum trabecular metal cones to fill the defect, followed by implantation of rotating hinge knee prosthesis. At an average follow-up of 57 months (4.75 years), the average Knee Society Score was 74.56 (64-87) and the mean range of motion was 97.8 (70-120) degrees. Radiologically, all 16 knees showed good osteointegration with no evidence of progressive radiolucency or loosening. Complications included two patients with superficial infection (healed with antibiotics), and one with recurrence of GCT requiring tumor megaprosthesis reconstruction. The authors concluded that the stacked configuration of cones provides additional coverage and stability with good osteointegration and found it to be particularly useful in distal femoral GCTs and fracture situations. They recommend the use of stacked cones in selected cases of severe distal femoral deficiency.
A revision total knee replacement (TKR) poses a challenge relating to management of bone defects, obtaining optimal limb alignment, recreation of joint line, achieving a stable construct to enable early mobilization, and ensuring precise soft tissue tension and flexion-extension gaps. ${ }^{1,2}$ Massive iatrogenic bone defect around the knee is also encountered after wide surgical resection of tumors, for example, giant cell tumors (GCTs), involving the epiphyseal-metaphyseal region of distal femur and proximal tibia. There has been a constant

received

May 10, 2018

accepted

July 23, 2018

published online

September 6, 2018 endeavor to develop newer techniques to deal with massive bone defects around the knee.

Severe distal femoral bone loss, sometimes extending up to the metaphysis and metaphyseo-diaphyseal junction (Anderson Orthopaedic Research Institute [AORI] types 2 and 3$)^{3}$ have been previously dealt with by using bulk cementing, impaction bone grafting, structural allografts or autografts, metal augments, trabecular metal (TM) tantalum cones, and sometimes requiring megaprosthesis reconstruction, depending on the
Copyright $\odot 2019$ by Thieme Medical Publishers, Inc., 333 Seventh Avenue, New York, NY 10001, USA. Tel: +1(212) 584-4662.
DOI https://doi.org/ 10.1055/s-0038-1669789. ISSN 1538-8506. 
size and location of the defect. ${ }^{4,5}$ The latter option is particularly relevant in cases with partial or severe loss of cortical bone extending up to the distal femoral diaphyseal region, as seen following tumor resection surgery, periprosthetic fractures, or severely comminuted distal femoral fractures. ${ }^{6}$

The wide metaphyseal region of distal femur offers a unique option in terms of reconstruction of these bone defects. Building up of severe bone defects with cement alone may cause reduced cement pressurization and lower cement-bone interdigitation. Structural/bulky and impaction bone grafting may not provide immediate stability, unpredictable graft incorporation besides the problems relating to allografts (procurement, storage, and transmission of infection), and donor site morbidity following harvesting of autografts. ${ }^{7}$

The introduction of porous metal tantalum TM cones as a mode of biological fixation serves to eliminate many of the potential disadvantages described above. ${ }^{8}$ Porous TM cones provide a new tool for modular reconstruction in type 2 and 3 defects (AORI) of the distal femur. The wider metaphyseal cross-section provides an optimal bed for circumferential contact, bony ingrowth, and neovascularization while using tantalum cones for reconstruction. But in cases with partial/ near complete loss of the condyles with extension up to and sometimes beyond the metaphyseo-diaphyseal region, achieving adequate metaphyseal filling and a press-fit stable implant construct with a single TM cone may sometimes be difficult due to longer and wider cross-section of the metaphyseal region. In such situations, the solitary distal femoral cone may not come in contact with an adequate rim of cortical/metaphyseal bone to provide adequate press-fit and bone-metal contact, which may necessitate concomitant use of structural allograft/autograft or megaprosthesis reconstruction.

To address this issue, we have used a stacked cone technique, where two cones are used together to address severe distal femoral bone loss in defects which would otherwise require use of structural bone grafts or megaprosthesis reconstruction. In this retrospective study, we present our experience and mid-term results of the stacked two-cone technique while dealing with severe distal femoral bone loss during revision TKR and following distal femoral fractures and tumor resection (of GCTs).

\section{Methods}

This is a retrospective study of 16 consecutive patients undergoing TKR and who required a stacked cone arrangement of two TM cones to deal with severe distal femoral bone loss. All cases were operated by senior consultant (A.R.) between March 2008 and July 2014 and had a minimum follow-up of 2 years. Institutional Review Board approval was taken prior to commencement of the study.

The clinical records of all patients were analyzed and the demographic data, etiology, indication for surgery, duration of symptoms, comorbidities, and surgical findings were noted. Preoperative mobility, range of motion (ROM), radiographic analysis with alignment, and component positioning were noted. The pre- and postoperative Knee Society Score ${ }^{9}$ (KSS) were recorded. Postoperative limb alignment, ROM, and KSS scores were recorded by senior physiotherapist at each follow-up visit at 6 weeks, 6 months, and half-yearly thereafter. Postoperatively, long leg weight-bearing X-rays were done to determine bony integration, alignment of the components, and to look for evidence of aseptic loosening.

\section{Surgical Technique}

A standard midline skin incision and medial parapatellar approach was used in every case. Additional exposure in the form of quadriceps snip was required in four cases. The technique of revision was standard in all cases including removal of any existing implant components with thorough local debridement while preserving the bone stock and avoiding injury to the extensor mechanism. Wide local excision of the distal femoral GCT was done after due preoperative planning and consultation with the oncology team.

The final bone defect was graded according to the AORI classification where the femoral and tibial defects are classified separately. ${ }^{3}$ The need for TM cones was assessed by determining the extent of bone defect and the seating of the trial cones and implant components. The distal femoral bed was prepared and contoured using a high-speed burr to ensure press-fit seating of the femoral TM cones. Sequential reaming of the medullary canal was done to determine the ideal femoral stem size.

TM cones (Zimmer, Warsaw, IN) serve as modular augments and are available in different sizes (small, medium, and large) and are of two types (metaphyseal and diaphyseal). The metaphyseal cones are available as asymmetric components with right and left varieties and a maximum height of $30 \mathrm{~mm}$, while the diaphyseal are symmetric. Hence, theoretically, up to $6 \mathrm{~cm}$ of distal femoral defect can be reconstructed using the stacked cone construct. Offset stems cannot be used and cemented fixation has been recommended while using with rotating hinge knee (RHK; Zimmer, Warsaw, IN) designs, as used in all of our cases. The maximum diameter of the straight stems that can be used is $16 \mathrm{~mm}$ for small and medium sizes and $17 \mathrm{~mm}$ for large variety.

The patients in our study presented with severe distal femoral bone loss extending up to the metaphyseo-diaphyseal junction with involvement of variable circumference and length of distal diaphysis (e.g., shown in - Fig. 1). A single TM cone could not provide adequate coverage of the defect and necessitated the use of two TM cones (metaphyseal + diaphyseal) skewered over a stem (cone-on-cone arrangement) as shown in - Fig. 2A and B. Such an arrangement was particularly useful in those with partial circumferential loss of the distal metaphyseo-diaphyseal region. As shown in -Fig. 2B, presence of a cross-section of the diaphyseal cortex enables proper seating of the second (proximal-most) TM cone adding stability to the metaphyseal-diaphyseal region, increasing cross-sectional area of bony contact and metaphyseal filling and providing added stability to the femoral component. 


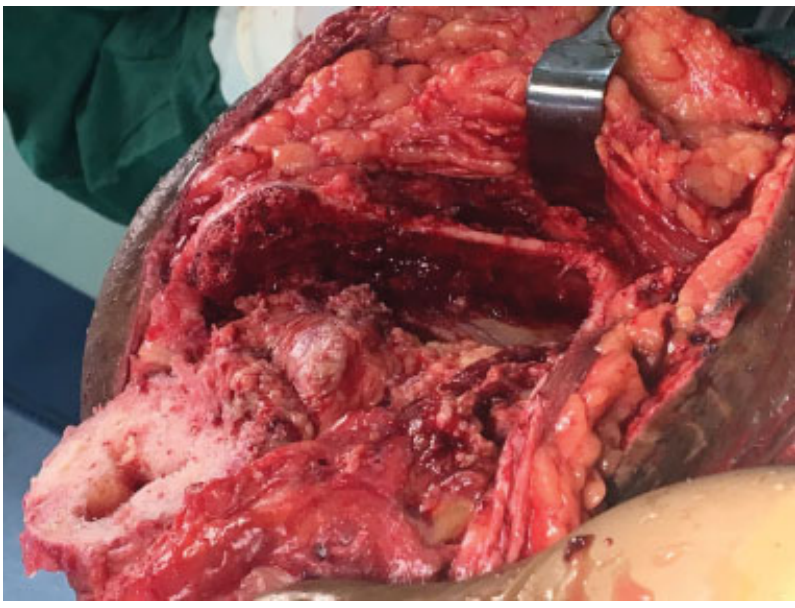

Fig. 1 Severe distal femoral bone loss with loss of medial condyle.

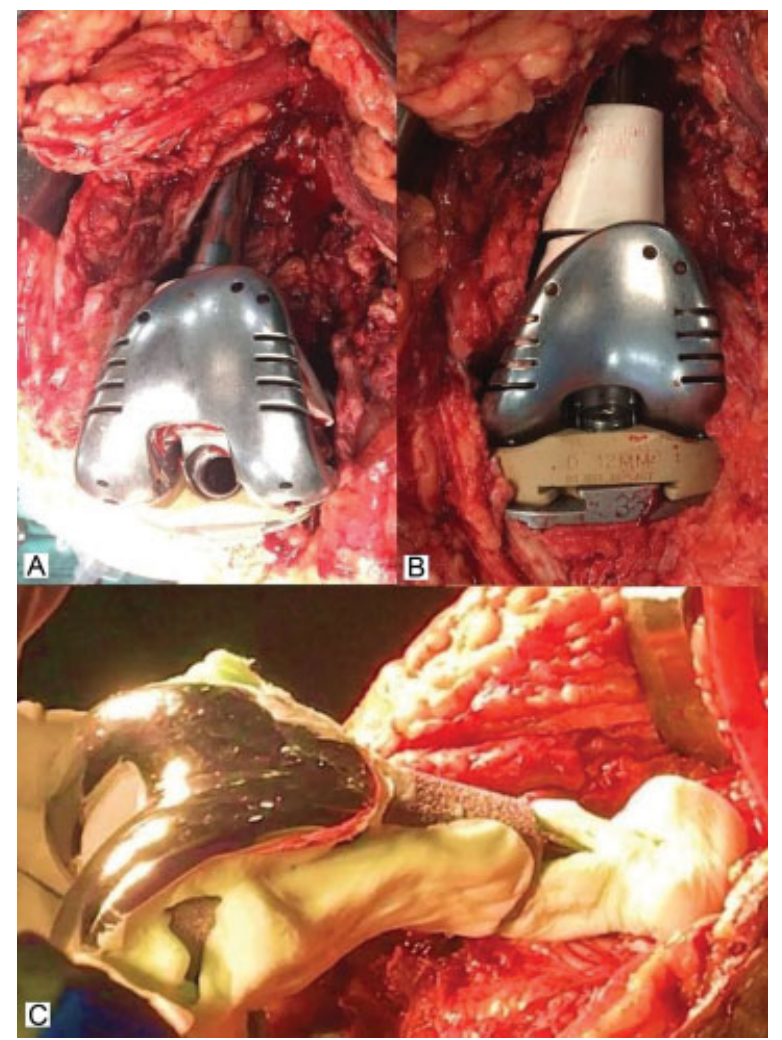

Fig. 2 (A) Incomplete coverage of defect with single metaphyseal cone following trial reduction. (B) Better coverage of bone defect could be achieved with two (metaphyseal + diaphyseal) distal femoral cones. (C) Final cemented implantation of femoral component with two stacked cones.

Final sizing of cones, femoral component, and stem was done using different sized trials. Fluoroscopic confirmation was done in all the cases after trial implantation to ensure optimal implant positioning and alignment. Cemented fixation was done and any gaps between the cones and bones were filled with morsellized autograft and/or allograft for augmentation of local bone stock ( $\mathbf{- F i g . 2 C )}$ ). An RHK design was used in all our cases. The uncovered parts of the cones exposed to local soft tissue were covered with cement to avoid irritation and inflammatory reaction in the surrounding tissue. Bone wax can also be used for this purpose. ${ }^{10}$

Immediate weight-bearing was started in 9 cases, while it was delayed for 6 weeks in 7 cases. A dial-lock brace was used postoperatively and assisted mobilization was initiated from postoperative day 1 followed by active ROM.

\section{Statistical Analysis}

The demographic characteristics of patients, functional scores, and ROM are reported as mean with range. Statistical significance of the difference in functional outcomes between preoperative and postoperative period was evaluated with paired $t$-test. Statistical Package for the Social Sciences (SPSS) version 20.0 was used. Statistical significance was defined at $p<0.05$.

\section{Results}

This is a retrospective study of 16 patients (16 knees) in which stacked two-cone technique was used for the management of severe distal femoral bone loss. Patient demographics and outcomes for management of severe femoral bone loss with dual TM cones are summarized in - Table 1. The average age at the time of surgery was 63.8 years (range: 42-84 years). There were 6 males and 10 females: 56.2\% (9/ 16 knees) had F3 type defect and 43.8\% (7/16 knees) had F2B type of bone loss. The main cause for bone loss in primary cases (31.25\%) was tumor (GCT, 4 knees) and comminuted distal femur fracture with severe osteoarthritis (1 knee), whereas in revision (68.75\%) was infection (5 knees), aseptic loosening (3 knees), and periprosthetic fracture (3 knees).

The average KSS improved significantly from 45.33 points (range: $24-74)$ preoperatively to 74.56 points (range: $64-87$ ) postoperatively $(p<0.001)$. The average ROM improved significantly from 52.9 degrees (range: $10-100$ ) preoperatively to 97.8 degrees (range: 70-120) postoperatively $(p<0.001)$. On radiological evaluation, all the cones appeared to be closely apposed to the host bone with radiological evidence of osteointegration at the site of bone trabecular interface on an average follow-up of 57 months. There was no evidence of implant loosening and migration of the implant at the latest follow-up.

Out of 18 patients in this study, one patient required second surgery due to recurrence of tumor 3.4 years after primary surgery for which tumor megaprosthesis was used after en bloc tumor resection. Two patients had superficial infection which subsided with oral antibiotics. At the time of explantation, the patient with recurrence of GCT showed excellent evidence of osteointegration of the two stacked cones (-Fig. $\mathbf{3}$ ).

\section{Discussion}

The use of the dual-stacked TM cone technique appears to be an extremely viable alternative to the use of structural bone grafts, metal augments usually with bulky cementation, and even to tumor megaprosthesis while dealing with larger uncontained defects (type 2B and 3 AORI) as encountered in 
Table 1 Patient demographics and outcomes following stacked two-cone technique for distal femoral reconstruction

\begin{tabular}{|c|c|c|c|c|c|c|c|c|c|c|}
\hline \multirow[t]{2}{*}{ S. no. } & \multirow[t]{2}{*}{ Age } & \multirow[t]{2}{*}{ Sex } & \multirow[t]{2}{*}{$\begin{array}{l}\text { Primary/ } \\
\text { Revision }\end{array}$} & \multirow[t]{2}{*}{ Diagnosis } & \multirow[t]{2}{*}{ Side } & \multirow[t]{2}{*}{$\begin{array}{l}\text { AORI } \\
\text { type }\end{array}$} & \multicolumn{2}{|l|}{ KSS } & \multicolumn{2}{|c|}{$\begin{array}{l}\text { ROM } \\
\text { (degrees) }\end{array}$} \\
\hline & & & & & & & Pre & Post & Pre & Post \\
\hline 1 & 42 & $M$ & Primary & GCT & $\mathrm{R}$ & F3 & 70 & 82 & 80 & 120 \\
\hline 2 & 53 & $M$ & Primary & GCT & $\mathrm{R}$ & F3 & 74 & 87 & 80 & 110 \\
\hline 3 & 72 & $\mathrm{~F}$ & Revision & Aseptic loosening & $\mathrm{L}$ & F2B & 55 & 67 & 60 & 95 \\
\hline 4 & 65 & $\mathrm{~F}$ & Revision & Periprosthetic \# & $\mathrm{L}$ & F3 & NR & 72 & NR & 90 \\
\hline 5 & 62 & $M$ & Revision & Septic loosening & $\mathrm{L}$ & F2B & 38 & 65 & 25 & 70 \\
\hline 6 & 84 & $M$ & Revision & Aseptic loosening & $\mathrm{L}$ & F2B & 32 & 64 & 60 & 90 \\
\hline 7 & 69 & $\mathrm{~F}$ & Revision & Aseptic loosening & $\mathrm{L}$ & F3 & 46 & 75 & 65 & 115 \\
\hline 8 & 57 & $\mathrm{~F}$ & Revision & Septic loosening & $\mathrm{L}$ & F2B & 41 & 74 & 20 & 110 \\
\hline 9 & 83 & $\mathrm{~F}$ & Revision & Periprosthetic \# & $\mathrm{R}$ & F3 & NR & 65 & NR & 80 \\
\hline 10 & 71 & $\mathrm{~F}$ & Revision & Periprosthetic \# & $\mathrm{L}$ & F3 & NR & 68 & NR & 100 \\
\hline 11 & 68 & $\mathrm{~F}$ & Revision & Septic loosening & $\mathrm{L}$ & F2B & 24 & 76 & 25 & 80 \\
\hline 12 & 65 & $\mathrm{~F}$ & Revision & Septic loosening & $\mathrm{R}$ & F2B & 29 & 75 & 10 & 90 \\
\hline 13 & 69 & $\mathrm{~F}$ & Primary & $\begin{array}{l}\text { Distal femur communited \# with } \\
\text { bone loss with severe osteoarthritis }\end{array}$ & $\mathrm{R}$ & F3 & NR & 80 & NR & 95 \\
\hline 14 & 46 & $M$ & Primary & GCT & $\mathrm{R}$ & F3 & 63 & 85 & 70 & 120 \\
\hline 15 & 51 & $M$ & Primary & GCT & $\mathrm{L}$ & F3 & 28 & 82 & 100 & 110 \\
\hline 16 & 64 & $\mathrm{~F}$ & Revision & Septic loosening & $\mathrm{R}$ & F2B & 44 & 76 & 40 & 90 \\
\hline
\end{tabular}

Abbreviations: AORI, Anderson Orthopaedic Research Institute; F, female; GCT, Giant cell tumor; KSS, Knee Society Score; L, left; M, male; NR, not reported; $R$, right; $R O M$, range of motion.

our study. All the cases showed good osteointegration with no evidence of radiolucency surrounding the implant components, excellent postoperative alignment, and significant improvement in outcome scores at an average follow-up of 57 months.

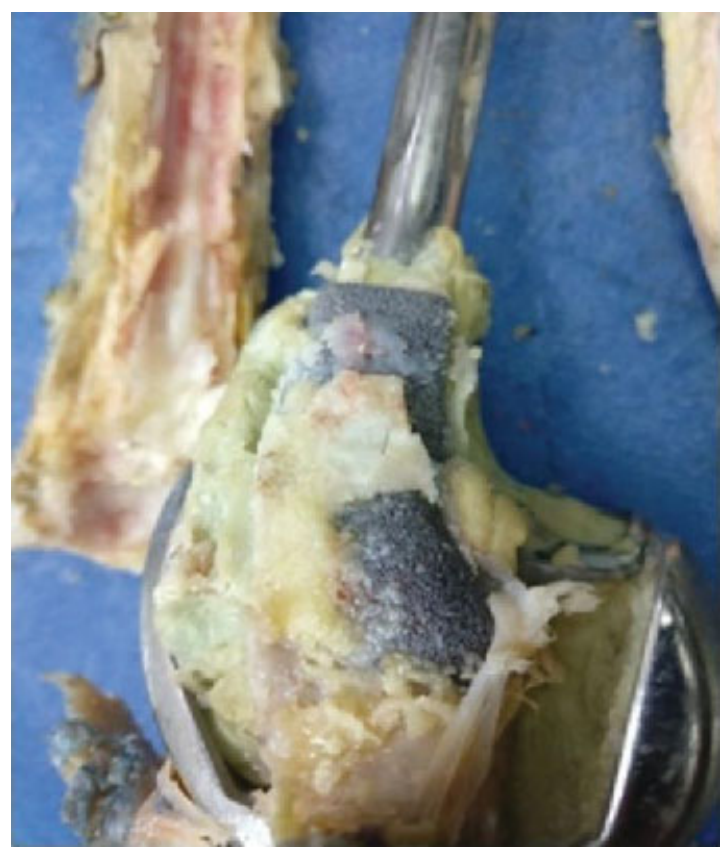

Fig. 3 Resected specimen of patient with recurrence of giant cell tumor treated with dual cones showing good osteointegration.
An important limitation of our study is a relatively small sample size. However, given the fact that all these cases are extreme conditions of bone loss with relatively rare incidences, this sample size was considered to be adequate. The strength of our study is that there is no loss in follow-up till date with an average follow-up of 57 months.

The optimal treatment method for addressing large femoral defects during knee replacement surgery has not been established. The recent addition of femoral porous tantalum TM cones has provided an alternative tool for addressing these defects. The many cited advantages of TM cones include high porosity with interconnected pores mimicking metaphyseal cancellous bone with negative charge which facilitates osteoblast-mediated bone ingrowth, low modulus of elasticity (lower stress shielding), and low bacterial adherence. ${ }^{11,12}$ The high porosity and high coefficient of friction allows for scratch fit providing immediate stability and allowing early weight-bearing. The ultraporosity additionally facilitates good cement interdigitation on the intramedullary side during cemented fixation. They are associated with lower incidence of component migration or subsidence as evaluated by radioisometric analysis, which may occur due to the depleted metaphyseal cancellous bone and during initial months of local bone resorption following the revision surgery. ${ }^{13}$ The osteoconductive and osteoinductive property of TM cones allows for good bony ingrowth as seen by the formation of bridging callus across the site of the defect aiding in the restoration of bone mass. Multiple studies have reported promising outcomes with the use of TM cones in revision TKR (-Table $\mathbf{2}$ ). 
Table 2 Literature review outcomes following use of trabecular metal cones in total knee arthroplasty

\begin{tabular}{|c|c|c|c|c|c|c|c|c|c|c|}
\hline \multicolumn{2}{|l|}{ Study } & $\begin{array}{l}\text { Long and } \\
\text { Scuderi }^{12}\end{array}$ & $\begin{array}{l}\text { Howard } \\
\text { et } \mathrm{al}^{14}\end{array}$ & $\begin{array}{l}\text { Lachiewicz } \\
\text { et al }\end{array}$ & $\begin{array}{l}\text { Schmitz } \\
\text { et al }{ }^{16}\end{array}$ & $\begin{array}{l}\text { Villanueva } \\
\text { et al }{ }^{17}\end{array}$ & $\begin{array}{l}\text { Derome } \\
\text { et al }\left.\right|^{18}\end{array}$ & $\begin{array}{l}\text { Boureau } \\
\text { et } \mathrm{al}^{19}\end{array}$ & $\begin{array}{l}\text { Girerd } \\
\text { et } \mathrm{al}^{20}\end{array}$ & $\begin{array}{l}\text { Our } \\
\text { study }\end{array}$ \\
\hline \multicolumn{2}{|l|}{ Year } & 2009 & 2011 & 2012 & 2013 & 2013 & 2014 & 2015 & 2016 & 2018 \\
\hline \multicolumn{2}{|l|}{ No. of knees } & 16 & 24 & 27 & 38 & 21 & 29 & 7 & 52 & 16 \\
\hline \multirow{2}{*}{$\begin{array}{l}\text { Tibia/Femur } \\
\text { implant }\end{array}$} & $T$ & 16 & 0 & 24 & 25 & 11 & 17 & 0 & 38 & 0 \\
\hline & $\mathrm{F}$ & 0 & 24 & 9 & 29 & 18 & 16 & $\begin{array}{l}7 \text { (two-cone } \\
\text { technique) }\end{array}$ & 34 & 16 \\
\hline \multirow{2}{*}{$\begin{array}{l}\text { Preop class } \\
\text { (AORI) }\end{array}$} & $2 \mathrm{~A} / \mathrm{B}$ & 5 & NR & 4 & 19 & 9 & NR & 2 & 43 & 6 \\
\hline & 3 & 11 & NR & 29 & 19 & 19 & NR & 5 & 39 & 10 \\
\hline \multicolumn{2}{|c|}{$\begin{array}{l}\text { Mean follow-up } \\
\text { (mo) }\end{array}$} & 31 & 33 & 39 & 37 & 36 & 33 & 17 & 25 & 57 \\
\hline \multicolumn{2}{|l|}{ Infection } & 2 & 0 & 1 & 0 & 2 & 2 & 0 & 4 & 2 \\
\hline \multicolumn{2}{|l|}{ Loosening } & 0 & 0 & 1 & 1 & 0 & 0 & 0 & 1 & 0 \\
\hline \multicolumn{2}{|l|}{ Reoperation } & 2 & 5 & 4 & 2 & 2 & 4 & 0 & 4 & 1 \\
\hline
\end{tabular}

Abbreviations: AORI, Anderson Orthopaedic Research Institute; NR, not reported.

The dual-stacked cone technique was previously described by Boureau et al in seven cases of severe distal femoral defects (two-F2B and five-F3 AORI) which included four aseptic and three septic revisions with an average follow-up of 17 months (12-25 months). The International Knee Society score ranged from 78 to 143 with authors reporting good alignment and osteointegration at last follow-up. ${ }^{19}$

Girerd et al used a similar two-cone technique in 12 cases among their series of 51 patients and though the details regarding demography, etiology, and functional outcomes among these 12 cases were not discussed separately, they reported overall good outcomes with no evidence of loosening in all these cases. ${ }^{20}$

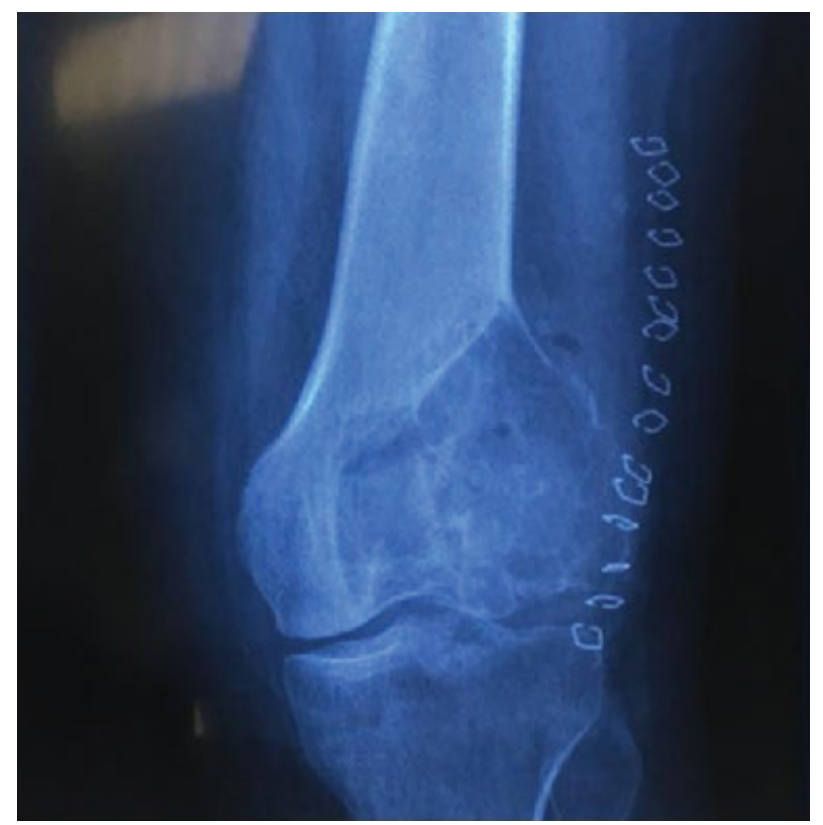

Fig. 4 Preoperative radiograph of distal femoral giant cell tumor.
We found this technique to be extremely useful when used in the management following wide surgical excision of distal femoral GCTs (-Figs. 4 and 5) and distal femoral fractures (primary or periprosthetic) (-Figs. 6 and 7). Good functional outcome scores and ROM were seen in all the cases (although recurrence of GCT was seen in 1 out of 4 cases). This method indeed may prove to be a promising option for the management of distal femoral GCTs requiring wide surgical excision and as an alternative to using bulky bone grafts or tumor megaprosthesis.

Among all the 35 cases (including those in our study) reported till date where a two-stacked cone technique has been used for metaphyseal/metaphyseo-diaphyseal filling of severe distal femoral defects (F2B and F3 AORI), all have reported good functional outcome, alignment, and good osteointegration without detection of radiolucencies in any patient during follow-up.

Another example of such an extended indication of use of tantalum cones has been described in a case report by McNamara et al where a custom-made cylindrical distal femoral TM cone (length $7.5 \mathrm{~cm}$ ) was used in a 55-year-old patient who had undergone three previous revision surgeries. $^{21}$ The implant was designed based on computed tomography scan images in collaboration with industry engineers. They reported good functional outcome and osteointegration at 2-year follow-up. Such "off-the-shelf" modifications of TM cones are unlikely to be universally available besides being expensive, and hence, use of twostacked cones can be the best available alternative that can provide a height of approximately $6 \mathrm{~cm}$ for distal femoral reconstruction.

A systematic review by Beckmann et al has compared the differences in outcomes in total knee arthroplasties with knees reconstructed with either bulk/structural allografts (551 knees) or TM cones (254 knees). Following use of bulk allografts, rate of loosening/fracture of allografts was $6.5 \%$ 


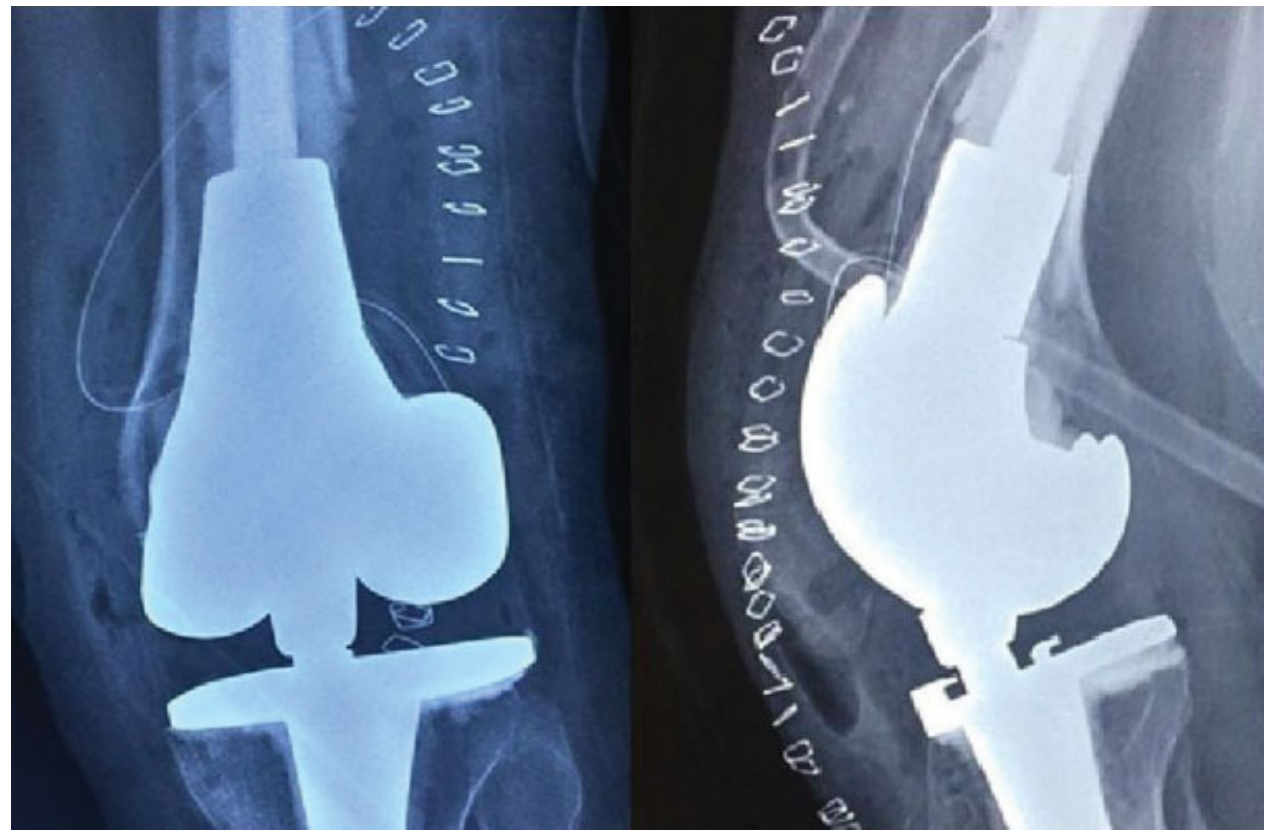

Fig. 5 Postoperative radiographs of giant cell tumor treated with stacked cones.

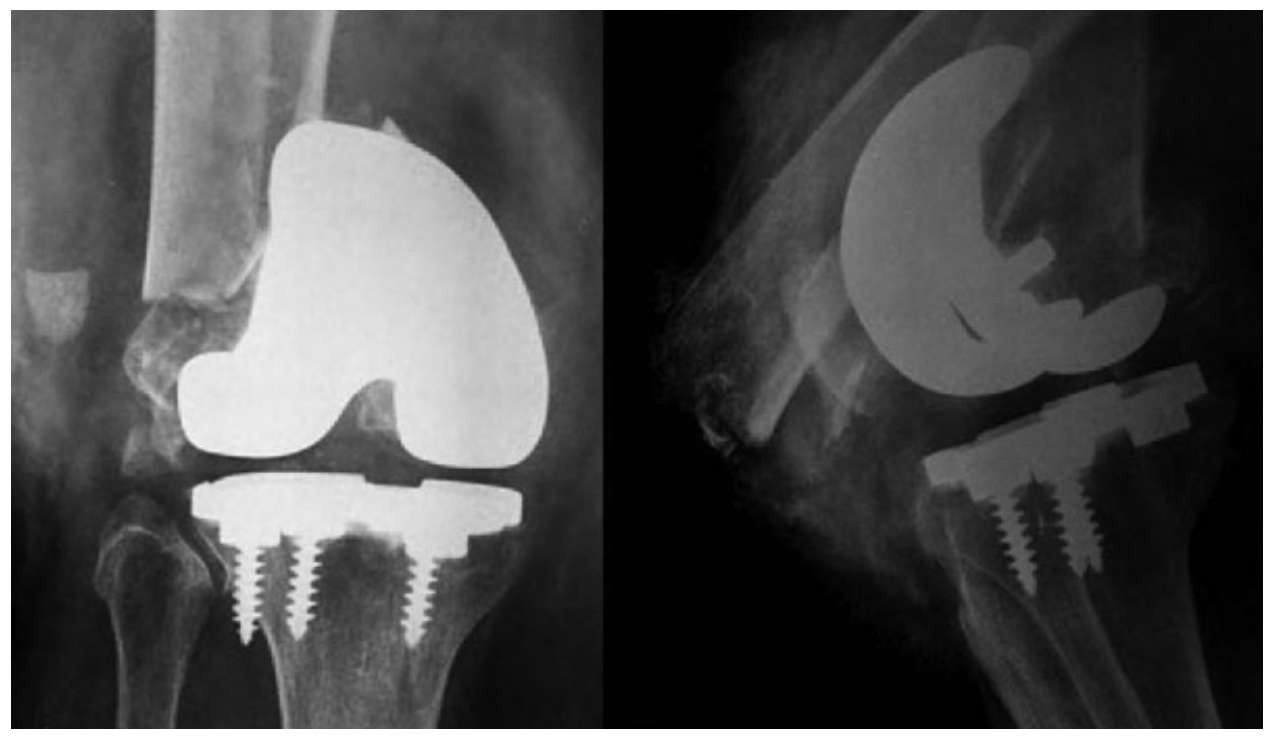

Fig. 6 Preoperative radiographs of periprosthetic fracture with severe comminution.

overall (0.9\% with TM cones), prosthetic loosening was $3.4 \%$ ( $0.9 \%$ with TM cones), and infection requiring revision was 5.5\% (2.2\% with TM cones). The estimated risk of loosening (odds ratio) with TM cones was one-fourth and of failure was half as compared with structural allografts. ${ }^{7}$ This shows a clear advantage of TM cones over structural allografts.

The reported failure rates of tumor megaprosthesis is high and may be up to 40 to $75 \%$ in some series ${ }^{22}$ with mechanical causes of failure accounting for up to $60 \%$ of the cases. ${ }^{23}$ This is in contrast to TM cones where the failure rates are higher during initial few months and the survival and mechanical stability is expected to get better over the years once good osteointegration is achieved.
An important pitfall of using this stacked cone technique is the risk of malposition of the stemmed femoral component, more so because of the inability to use offset stems in such situations. Intraoperative fluoroscopic/radiographic evaluation after placement of trial components should be done in all the cases. Also, the use of a smaller diameter cemented stem into larger cone can allow some degree of manipulations to be made for suitable positioning of the implant. The cost of another additional TM cone may be a limitation, though this option is less expensive than tumor megaprosthesis. Considering the unpredictability and risks involved with the use of structural allografts, use of stacked cones may eventually prove to be a cost-effective strategy and further studies are 


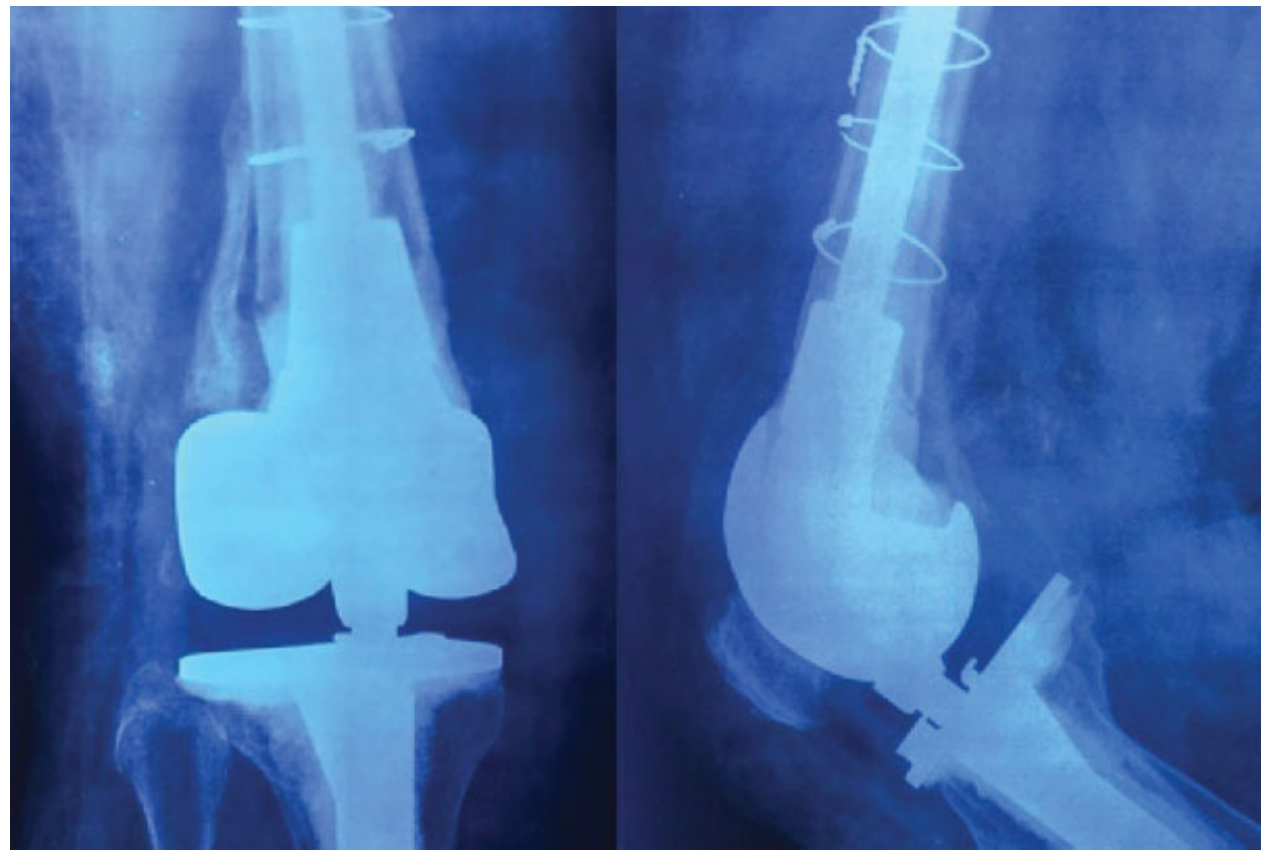

Fig. 7 Postoperative radiographs of periprosthetic fracture treated with stacked cones showing good osteointegration and bridging callus formation.

required in this regard. A major problem with use of TM cones is the technical challenges faced during implant removal in case of failure due to infection or, as seen in our case, recurrence of tumor, which may eventually lead to extensive bone loss during revision or en bloc resection requiring megaprosthesis reconstruction. This fact should be kept in mind and explained to the patients. Further studies and follow-up are required to evaluate the long-term survivorship of this two-cone technique.

\section{Conclusion}

The results following stacked two-cone arrangement of TM cones while dealing with distal femoral bone defects have been consistently good and predictable. Such an arrangement can be used as an extended indication while dealing with primary replacements such as distal femoral GCTs and during complex revision scenarios. We recommend this technique as an extremely viable alternative to use in place of structural allografts and megaprosthesis reconstruction during management of severe distal femoral defects.

\section{Funding \\ None.}

\section{Conflict of Interest}

None.

\section{References}

1 Mancuso F, Beltrame A, Colombo E, Miani E, Bassini F. Management of metaphyseal bone loss in revision knee arthroplasty. Acta Biomed 2017;88(2-S):98-111
2 Panegrossi G, Ceretti M, Papalia M, Casella F, Favetti F, Falez F. Bone loss management in total knee revision surgery. Int Orthop 2014; 38(02):419-427

3 Engh GA, Ammeen DJ. Bone loss with revision total knee arthroplasty: defect classification and alternatives for reconstruction. Instr Course Lect 1999;48:167-175

4 Vasso M, Beaufils P, Cerciello S, Schiavone Panni A. Bone loss following knee arthroplasty: potential treatment options. Arch Orthop Trauma Surg 2014;134(04):543-553

5 Rorabeck CH, Smith PN. Results of revision total knee arthroplasty in the face of significant bone deficiency. Orthop Clin North Am 1998;29(02):361-371

6 Pala E, Trovarelli G, Angelini A, Maraldi M, Berizzi A, Ruggieri P. Megaprosthesis of the knee in tumor and revision surgery. Acta Biomed 2017;88( 2 -S):129-138

7 Beckmann NA, Mueller S, Gondan M, Jaeger S, Reiner T, Bitsch RG. Treatment of severe bone defects during revision total knee arthroplasty with structural allografts and porous metal conesa systematic review. J Arthroplasty 2015;30(02):249-253

8 Kim EG, Patel NK, Chughtai M, et al. Tantalum cones in revision total knee arthroplasty. J Knee Surg 2016;29(08):621-626

9 Insall JN, Dorr LD, Scott RD, Scott WN. Rationale of the Knee Society clinical rating system. Clin Orthop Relat Res 1989;(248): 13-14

10 Moore RE, Austin MS. Use of porous tantalum cones in revision total knee arthroplasty. Oper Tech Orthop 2012;22(04):209-221

11 Levine BR, Sporer S, Poggie RA, Della Valle CJ, Jacobs JJ. Experimental and clinical performance of porous tantalum in orthopedic surgery. Biomaterials 2006;27(27):4671-4681

12 Long WJ, Scuderi GR. Porous tantalum cones for large metaphyseal tibial defects in revision total knee arthroplasty: a minimum 2-year follow-up. J Arthroplasty 2009;24(07):1086-1092

13 Jensen CL, Petersen MM, Schrøder HM, Flivik G, Lund B. Revision total knee arthroplasty with the use of trabecular metal cones: a randomized radiostereometric analysis with 2 years of follow-up. J Arthroplasty 2012;27(10):1820-1826

14 Howard JL, Kudera J, Lewallen DG, Hanssen AD. Early results of the use of tantalum femoral cones for revision total knee arthroplasty. J Bone Joint Surg Am 2011;93(05):478-484 
840 Stacked Tantalum Cones as a Method for Treating Severe Distal Femoral Bone Deficiency Rajgopal et al.

15 Lachiewicz PF, Bolognesi MP, Henderson RA, Soileau ES, Vail TP. Can tantalum cones provide fixation in complex revision knee arthroplasty? Clin Orthop Relat Res 2012;470(01):199-204

16 Schmitz HC, Klauser W, Citak M, Al-Khateeb H, Gehrke T, Kendoff D. Three-year follow up utilizing tantal cones in revision total knee arthroplasty. J Arthroplasty 2013;28(09):1556-1560

17 Villanueva-Martínez M, De la Torre-Escudero B, Rojo-Manaute JM, Ríos-Luna A, Chana-Rodriguez F. Tantalum cones in revision total knee arthroplasty. A promising short-term result with 29 cones in 21 patients. J Arthroplasty 2013;28(06):988-993

18 Derome P, Sternheim A, Backstein D, Malo M. Treatment of large bone defects with trabecular metal cones in revision total knee arthroplasty: short term clinical and radiographic outcomes. J Arthroplasty 2014;29(01):122-126

19 Boureau F, Putman S, Arnould A, Dereudre G, Migaud H, Pasquier G. Tantalum cones and bone defects in revision total knee arthroplasty. Orthop Traumatol Surg Res 2015;101(02):251-255
20 Girerd D, Parratte S, Lunebourg A, et al. Total knee arthroplasty revision with trabecular tantalum cones: preliminary retrospective study of 51 patients from two centres with a minimal 2-year follow-up. Orthop Traumatol Surg Res 2016;102(04): 429-433

21 McNamara CA, Gösthe RG, Patel PD, Sanders KC, Huaman G, Suarez JC. Revision total knee arthroplasty using a custom tantalum implant in a patient following multiple failed revisions. Arthroplast Today 2016;3(01):13-17

22 Pala E, Trovarelli G, Calabrò T, Angelini A, Abati CN, Ruggieri P. Survival of modern knee tumor megaprostheses: failures, functional results, and a comparative statistical analysis. Clin Orthop Relat Res 2015;473(03):891-899

23 Capanna R, Scoccianti G, Frenos F, Vilardi A, Beltrami G, Campanacci DA. What was the survival of megaprostheses in lower limb reconstructions after tumor resections? Clin Orthop Relat Res 2015;473(03):820-830 